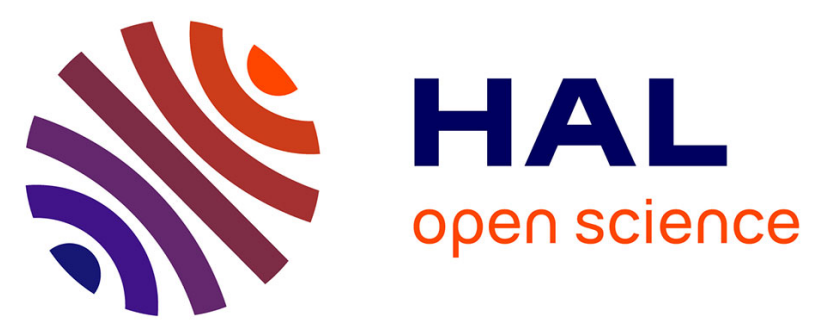

\title{
Origin of the super-enhanced light transmission through a 2-D metallic annular aperture array: a study of photonic bands
}

\author{
F. Baida, Van Labeke D, G. Granet, A. Moreau, A. Belkhir
}

\section{To cite this version:}

F. Baida, Van Labeke D, G. Granet, A. Moreau, A. Belkhir. Origin of the super-enhanced light transmission through a 2-D metallic annular aperture array: a study of photonic bands. Applied Physics B - Laser and Optics, 2004, 79 (1), pp.1-8. 10.1007/s00340-004-1497-3 . hal-00095069

\section{HAL Id: hal-00095069 \\ https://hal.science/hal-00095069}

Submitted on 22 Apr 2021

HAL is a multi-disciplinary open access archive for the deposit and dissemination of scientific research documents, whether they are published or not. The documents may come from teaching and research institutions in France or abroad, or from public or private research centers.
L'archive ouverte pluridisciplinaire HAL, est destinée au dépôt et à la diffusion de documents scientifiques de niveau recherche, publiés ou non, émanant des établissements d'enseignement et de recherche français ou étrangers, des laboratoires publics ou privés. 


\section{Origin of the super-enhanced light transmission through a 2-D metallic annular aperture array: a study of photonic bands}

\author{
F.I. BAIDA ${ }^{1, \infty}$ \\ D. VAN LABEKE ${ }^{1}$ \\ G. GRANET ${ }^{2}$ \\ A. MOREAU ${ }^{2}$ \\ A. BELKHIR ${ }^{3}$
}

\author{
${ }^{1}$ Département d'Optique P.M. Duffieux, Institut FEMTO-ST UMR 6174 CNRS \\ Université de Franche-Comté, 25030 Besançon Cedex,France \\ ${ }^{2}$ Lasmea, CNRS UMR 6602, Université Blaise-Pascal, 24 Avenue des Landais 63177 Aubière cedex, France \\ ${ }^{3}$ Laboratoire de Physique et Chimie Quantique, Université Mouloud Mammeri, Tizi-Ouzou, Algeria
}

ABSTRACT Recently a new structure showing a super-enhanced transmission [Optics Commun. 209, 17-22 (2002); Phys. Rev. B, 67, 155314, (2003)] has been proposed. The origin of this phenomenon was not clearly explained. In this paper, by using a numerical Order-N FDTD spectral method, we study the eigenmodes, the band structure and the dispersion curves of a photonic 2-D crystal made with coaxial circular cavities and made from a real metal. We show that the super-enhanced transmission of the finite structure is due to a cavity resonance of a single guided mode. An extensive characterization of this mode is presented, in terms of the spatial mode structure and effective index dispersion curve.

\section{1}

\section{Introduction}

In 1998, T. Ebbesen [1] published a very interesting paper showing enhanced transmission of light through a subwavelength circular aperture array in a metallic film. That paper has stimulated many studies on this subject. A short bibliography can be found in reference [2]. In most studies, structures showing an enhanced transmission are 1-D lamellar gratings [3] or 2-D arrays made of cylindrical cavities in the metallic film [4]. For such a 2-D structure, the transmission is significantly enhanced but remains small: experimentally an $8 \%$ zero order transmission is observed through a circular hole grating in gold (hole diameter $200 \mathrm{~nm}$, lattice constant $600 \mathrm{~nm}$, metal thickness $250 \mathrm{~nm}$ [4]).

Recently we have proposed a new structure $[2,5]$ which can exhibit a very large transmission. FDTD (finite difference in the time domain) calculations have demonstrated an $80 \%$ transmission in the visible region by using a 2-D grating made of coaxial cavities. As reported in reference [2], we studied the influence of the physical parameters on the transmission spectra of this annular aperture array (AAA). In reference [5], we calculated the near-field and field in the cavities. Examples of spectra are presented in Fig. 1 for two metal thicknesses [2].

The FDTD code, used to theoretically study the phenomenon, has been seriously and extensively checked by

Fax: +33-381-666-423, E-mail: fbaida@univ-fcomte.fr

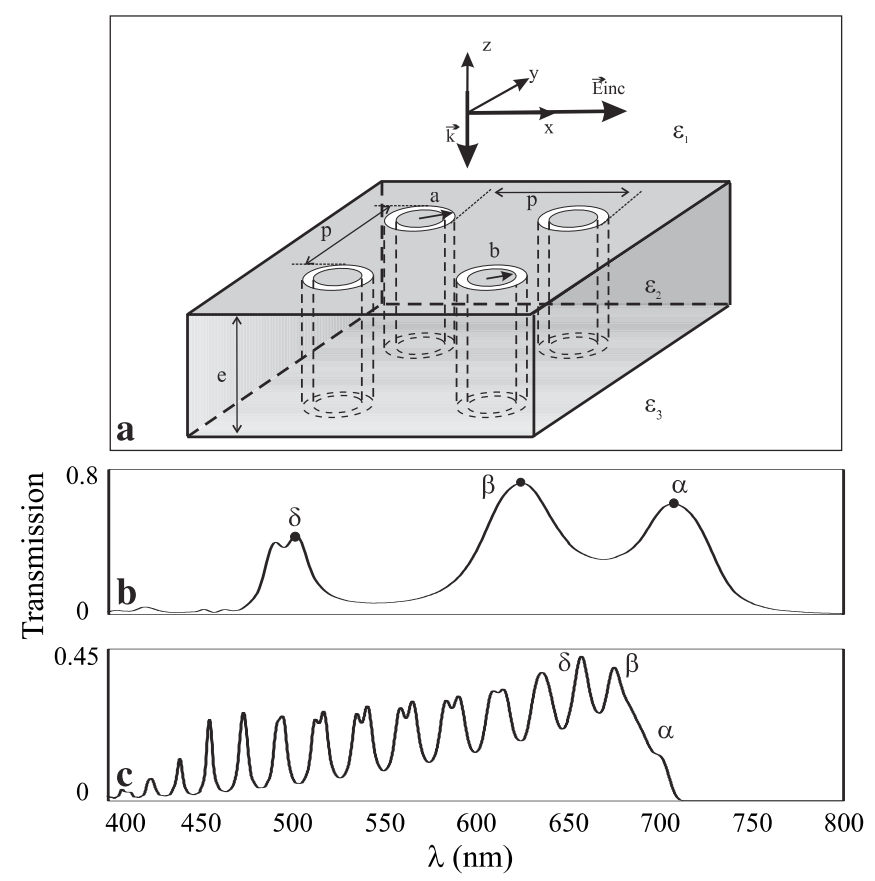

FIGURE 1 Zero order transmission efficiencies for AAA silver square grating structure (grating period $p=300 \mathrm{~nm}, a=75 \mathrm{~nm}$ and $b=50 \mathrm{~nm}$ in Fig. 2). a Schematic of the structure. In $\mathbf{b}$ the metal thickness is set to $e=300 \mathrm{~nm}$, in $\mathbf{c} e=1800 \mathrm{~nm}$

comparison with published results for similar structures. Moreover, a structure made with a 2-D array of square coaxial cavities has been studied with our code and with a RCWM code (rigorous coupled wave method) [6]. The results are plotted in Fig. 2 of that reference. The square coaxial structure also exhibits a very large transmission, and the two theoretical methods lead to similar transmission efficiencies (the small discrepancies between the two are probably created by meshing approximations in the FDTD method).

So, it has been theoretically demonstrated that a coaxial structure can exhibit a very large transmission, and experiments that investigate this effect are in progress. AAA could have the same applications that Ebbesen's structures have, but with a larger transmission efficiency and a better peak structure (see Fig. 1). Also, they can be used in near-field optical microscopy [7] to design optical modulators or tun- 
able filters [8] or to perform photolithography at a submicron scale [9]. It has also been proposed as having a potential application in flat-panel displays [10].

However, the interpretation of the large transmission obtained with AAA is not completely clear. All of the modes of a cylindrical waveguide made with a perfect metal have a cutoff. Consequently, when the radius is very small, the modes are evanescent and transmission of the aperture array remains small. A coaxial waveguide made with a perfect metal has a TEM mode (electro transverse magnetic) deprived of cut-off and we thought, at the beginning of our study, that this mode could explain the very large transmission of the AAA. But the field structure in the cavities does not look like the radial structure of a TEM mode.

So, the aim of this work is to study the mode structure in a grating of coaxial cavities. The studied structures are 2-D periodic gratings, infinite in the third direction. They can be considered as metallic photonic structures. Many papers are devoted to the theoretical study of metallic photonic crystals. It is difficult to cite all published works on this subject. We only mention a recent paper by Sakoda et al. [11] where a bibliography on the various theoretical methods can be found and is briefly discussed. Among all of the theoretical schemes, the FDTD method is a very efficient tool for obtaining the band structure of photonic crystals made with absorbing and dispersive material [12]. However, to our knowledge, the band structure of a photonic metallic crystal with coaxial cavities has not been studied.

This paper is therefore constructed around four sections: the first one introduces the notations and it briefly describes the computing scheme. In the second section, we study the band structure of a photonic crystal made of coaxial cavities, made from a perfect metal. This second section is needed to test our computing method and to check the location of the known modes of such a structure. In the third section, we replace the unrealistic perfect metal by silver. We determine the modes and we locate ones that could explain the very large transmission in the case of the AAA structure. Finally, we discuss the obtained results.

\section{$2 \quad$ Notations and computational principle}

The geometry and the parameters of the studied structures are indicated in Fig. 2. They are infinite in the $z$ direction. We denote the 2-D vectors in the transverse plane by capital letters: $\boldsymbol{R}=(x, y), \boldsymbol{K}=\left(k_{x}, k_{y}\right)$. In this paper, we only consider a coaxial structure with circular cross-sections, which corresponds to an annular cavity with an external radius $a$ and inner radius $b$. For the array, the coaxial cavities are periodic in the $x-y$ plane: it is a square grating of period $p$.

For the electromagnetic modes studied in this paper, $z$ is the propagation direction. A TE mode (transverse electric) has no $z$ component for the electric field $\left(E_{z}=0\right)$. For a TM mode (transverse magnetic), $H_{z}=0$, and for the TEM mode $H_{z}=E_{z}=0$.

For any mode, all of the field components contain a term proportional to $\mathrm{e}^{-\mathrm{i}\left(\omega t-k_{z} z\right)}$ where $\omega$ is the eigenfrequency and $k_{z}$ the propagating constant. The effective index of a mode, $\tilde{n}$, is directly connected to $k_{z}: k_{z}=\widetilde{n} \frac{\omega}{c}=\widetilde{n} \frac{2 \pi}{\lambda}$, where $c$ and $\lambda$ are respectively the speed of light and the wavelength in vacuum.

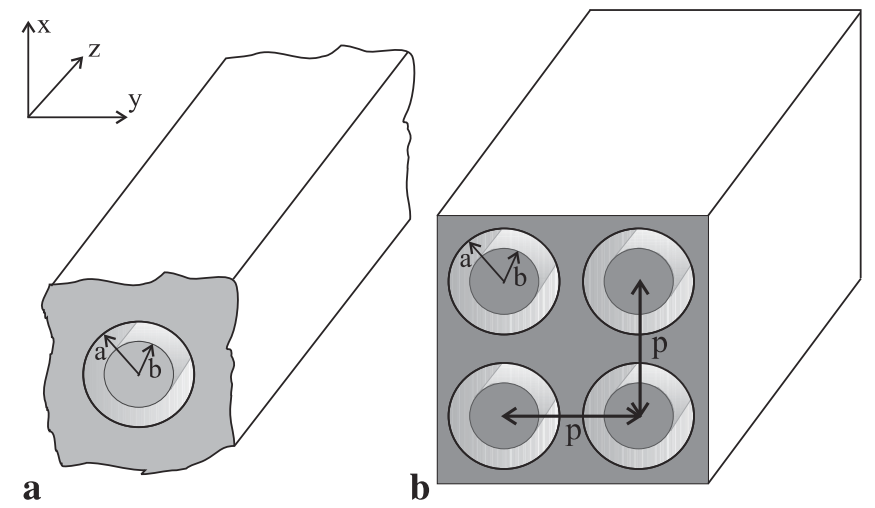

FIGURE 2 Coaxial waveguide geometry. a Single perfectly metallic waveguide, b Periodic grating of infinite coaxial waveguides made in silver metal

For the grating structure, the field amplitudes of each mode must also verify the Bloch theorem, and the electric field of one mode can be written:

$\boldsymbol{E}_{m}(\boldsymbol{R}, z, t)=\mathrm{e}^{-\mathrm{i}\left(\omega_{m} t-k_{z} z\right)} \sum_{\boldsymbol{G}} \boldsymbol{E}_{m}(\boldsymbol{G}) \mathrm{e}^{\mathrm{i}(\boldsymbol{K}+\boldsymbol{G}) \cdot \boldsymbol{R}}$

where $\boldsymbol{K}$ is a Bloch vector and $\boldsymbol{G}$ is a vector of the reciprocal grating.

In the FDTD, space and time are discretized. We use the "leapfrog" scheme proposed by Yee, which leads to a very efficient computing scheme when stability criteria are respected [13]. Electric and magnetic fields are calculated on interleaved grids; each component of the fields being at a different node of the grids. We take into account the dispersion and the absorption of the metal. The dielectric constant is therefore described by a simple Drude model that is very efficient in the studied spectral domain (visible and near infrared) [2].

To determine the mode structure of a photonic crystal, the plane wave expansion method (PWE) is generally used. However, the simple method does not work for a structure with absorption because the linear system is no longer hermitian. Our theoretical model is therefore based on the Order-N FDTD numerical method, developed in an interesting paper by Chan et al. [14]. The principle of the method is easily exposed: it consists of exciting the structure using an initial field whose spectrum extends over all of the possible frequencies of the structure modes. Then the FDTD code determines the field variations versus time. After a transient delay, only the eigenmodes of the structure persist and they lead to a great spectral radiant intensity at every eigenfrequency. The components of the initial electromagnetic field which do not correspond to an eigenmode will disappear.

The initial condition, defined by the fields at $t=0$, is given in Sect. III of [14]. The reciprocal vectors of the primitive unit cell were used in order to make sure that the initial field has nonzero projections onto all of the eigenstates of the studied structure. At the end of the FDTD calculation, a Fourier transform of the temporal response is calculated and leads to a spectrum composed of thin spectral lines corresponding to the eigenfrequencies (see the solid line in Fig. 3 for example). The spectral intensities are calculated at some randomly chosen nodes in the structure (30 nodes in our case) 


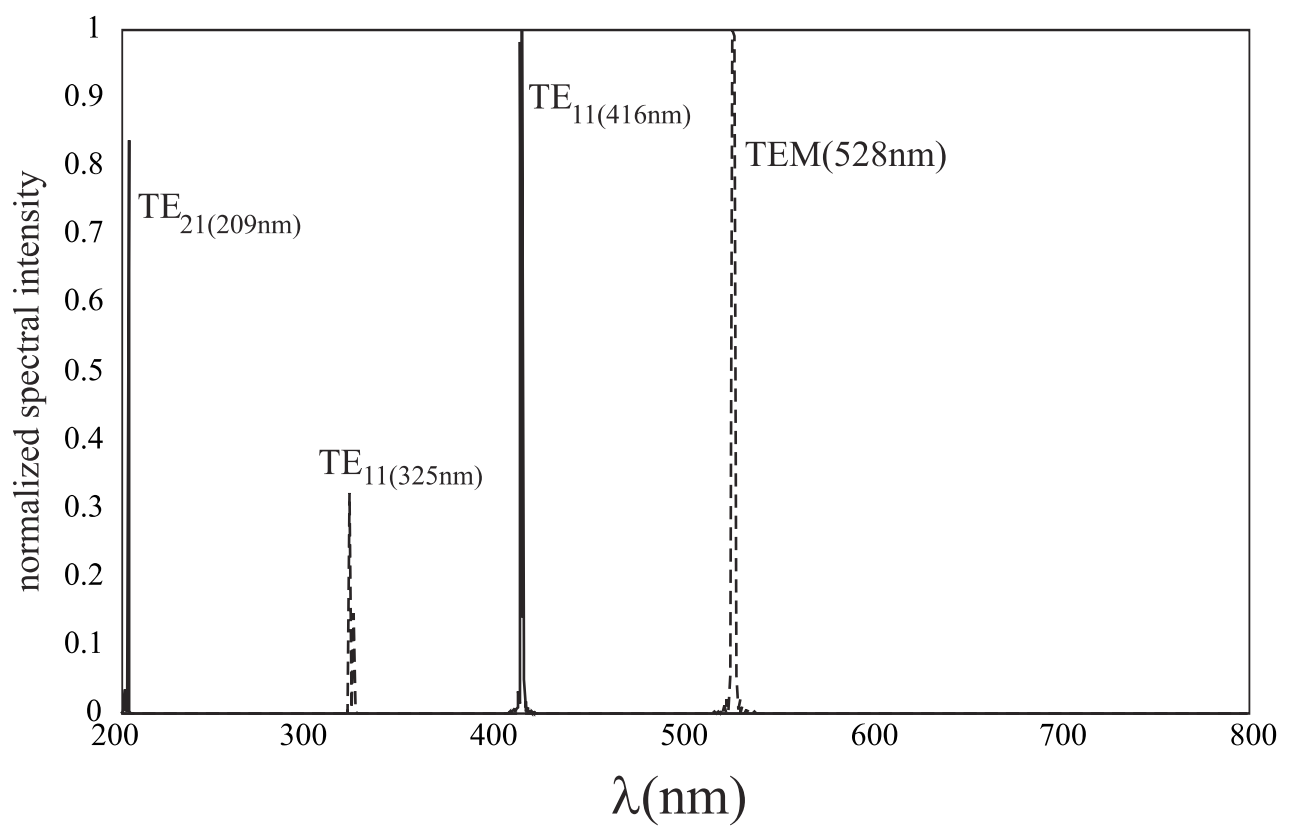

FIGURE 3 Normalized spectral intensities for two different $k_{z}$ values in the case of a perfectly metallic coaxial waveguide. $k_{z}=0$ for the solid line and $k_{z}=\frac{2 \pi}{500} \mathrm{~nm}^{-1}$ for the dashed line

in order to collect all of the modes. Notice that, for such a spectrum, the amplitude of the spectral lines has no physical meaning since it depends on the exact structure of the initial field.

By changing the values of $k_{z}$ and those of the Bloch vector $\boldsymbol{K}$, we can determine the dispersion relations of every mode: $\omega$ versus $k_{z}$ for a waveguide and $\omega$ versus $k_{z}$ and $\boldsymbol{K}$ for a grating structure.

In the case of a 2-D structure, there are two ways of writing the boundary conditions:

- When we attempt to point out the in-plane modes $\left(k_{z}=0\right)$, TE and TM modes can be treated separately by 2-D FDTD calculations. Periodic boundary conditions are imposed on both the two fields $\boldsymbol{E}$ and $\boldsymbol{H}$ in the $x$ and $y$ directions. No absorbing conditions are necessary here.

- To determine the out-of-plane modes, with $k_{z} \neq 0$, we should use a complete 3-D FDTD code and therefore add a third boundary condition along $z$ which takes into account the mode's propagation. However, a very small number of nodes in the $z$-direction is necessary and the boundary conditions in this direction are: $\Psi\left(x, y, z_{1}\right)=$ $\Psi\left(x, y, z_{2}\right) \times \mathrm{e}^{\mathrm{i} \cdot k_{z} \cdot\left(z_{2}-z_{1}\right)}$ where $\Psi$ is one component of the electric and/or the magnetic field.

In all of the studied cases, our FDTD lattice consists of one period $p$ of the grating. The spatial mesh steps were set to $\Delta_{x}=\Delta_{y}=\Delta_{z}=\frac{p}{120}$ and the temporal step to $\Delta_{t}=0.003 \frac{p}{c}$ as in [14]. The simulations used a total of 200000 time steps.

\section{Dispersion relations and mode structure for a perfect metal}

For a photonic crystal made in a perfect metal, the fields are thoroughly confined in the cavities and there is no coupling between the various cavities. Consequently, modes of the grating are simply connected to the mode of one cavity: the dispersion relations and the eigenfrequencies depend on $k_{z}$ and are independent of the Bloch vector. Such a structure is a very efficient test for our FDTD code.
The eigenmodes of a coaxial waveguide made in a perfect metal are well-known [15]. There is a $\mathrm{TEM}_{0}$ mode without cutoff. Its effective index is always $\widetilde{n}=1$ and it has cylindrical symmetry. The electric field of the $\mathrm{TEM}_{0}$ mode is radial.

Other propagating modes of a coaxial waveguide are well known. The dispersion relation of a mode verifies the relation:

$k_{z}^{2}=\frac{4 \pi^{2}}{\lambda^{2}}-\frac{4 \pi^{2}}{\lambda_{c}^{2}}$

where $\lambda_{c}$ is the cutoff wavelength of the mode. This cutoff is theoretically given by:

$\lambda_{c T M m, q} \approx \frac{2(b-a)}{q} \quad$ for TM modes,

$\lambda_{c T E m, 1} \approx \frac{\pi(a+b)}{m} \quad$ and

$\lambda_{c T E m, q} \approx \frac{2(b-a)}{q-1} \quad$ for TE modes.

where $a$ and $b$ are the outer and the inner radii of the coaxial waveguide.

We have determined the eigenmodes for an array of coaxial cavities with $a=75 \mathrm{~nm}$ and $b=50 \mathrm{~nm}$. Geometrical parameters of the structure were chosen in accordance with the ones of the silver AAA which exhibits a very large transmission. The normalized spectral intensities versus $\lambda$, calculated for two different values of $k_{z}$, are shown in Fig. 3 .

We have verified that the eigenfrequencies are independent of $\boldsymbol{K}$ which is an obvious result for a perfect metal, which implies a localisation of the fields in the cavities.

The solid line in Fig. 3 corresponds to $k_{z}=0$ (to the inplane modes). In this case, the eigenwavelengths are equal to the cutoff wavelengths of the coaxial waveguide $\left(\lambda_{\text {mode }}=\lambda_{c}\right.$ in (3) and (4)).

Only two modes appear between $\lambda=200 \mathrm{~nm}$ and $\lambda=$ $800 \mathrm{~nm}$. The two peaks are located at $\lambda=209 \mathrm{~nm}$ and 

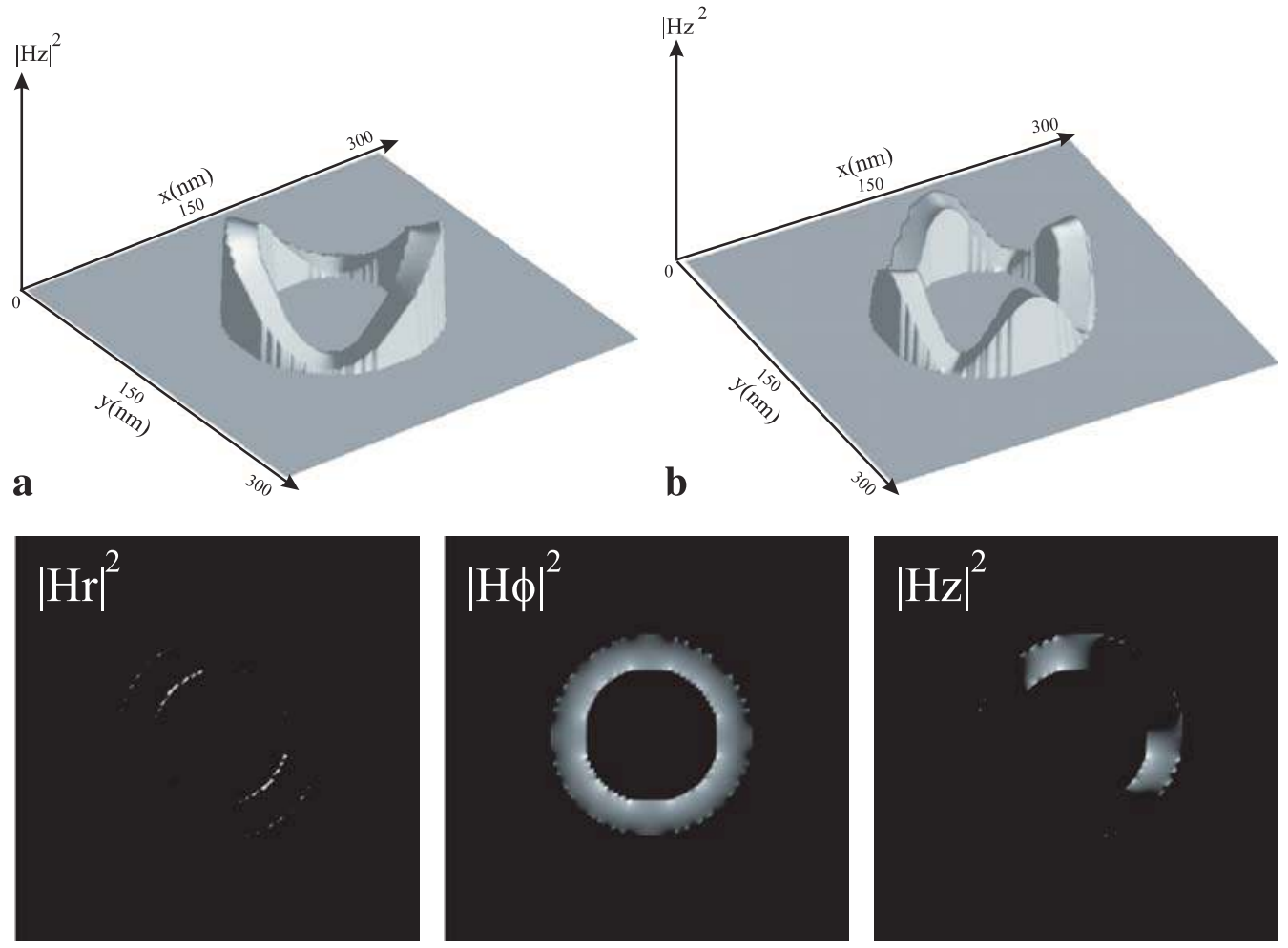

FIGURE $4|h z|^{2}$ of the two modes of a perfectly metallic coaxial waveguide for $k_{z}=0$. a For the $\mathrm{TE}_{11}$ mode, and $\mathbf{b}$ for the $\mathrm{TE}_{21}$ mode $\lambda=416 \mathrm{~nm}$. This is in accordance with the values of the $\mathrm{TE}_{21}$ and $\mathrm{TE}_{11}$ modes of (4) respectively. TM modes are not visible because their cutoff wavelengths are lower than $50 \mathrm{~nm}$.

The dashed line of Fig. 3 corresponds to $k_{z}=\frac{2 \pi}{500} \mathrm{~nm}^{-1}$. The shift of the two peaks is in good agreement with (2) and a third peak appears at $\lambda=528 \mathrm{~nm}$. This peak probably corresponds to the TEM mode. Its effective index is theoretically equal to 1 ; therefore it must be located at $500 \mathrm{~nm}$ instead of $528 \mathrm{~nm}$. This discrepancy is probably due to the spatial meshing of the object, which leads to a non-perfectly circular waveguide. The spatial step of the grid used in the FDTD calculation is $\Delta=2.5 \mathrm{~nm}$. It induces a staircase structure of the edges of the cavities which could be described by an uncertainty of the radii. Note here that such a mode (the TEM one) only exists when $k_{z} \neq 0$.

To confirm the nature of the modes of the solid line in Fig. 3, we present in Fig. 4 the square modulus of the magnetic field for both the two eigenwavelengths.

We note that, as is well-known, the $\mathrm{TE}_{11}$ mode's (Fig. 4a) $|h z|^{2}$ varies with respect to $\cos ^{2}(\varphi)$ while the $|h z|^{2}$ of the $\mathrm{TE}_{21}$ mode varies as $\cos ^{2}(2 \varphi)$ (Fig. 4b), where $\varphi$ is the azimuthal angle.

We also present in Fig. 5 the square modulus of the spatial distribution for the three cylindrical components $\left(H_{r}, H_{\varphi}\right.$ and $H_{z}$ ) of the magnetic field of the TEM out-of-plane mode (at $\lambda=528 \mathrm{~nm}$ ) corresponding to the third peak of the dashed line in Fig. 3.

Notice the cylindrical symmetry of the tangential component, $H_{\varphi}$. Theoretically, for a $\mathrm{TM}_{0}$ mode the other components must vanish: $H_{r}=H_{z}=0$. In our calculation, they do not vanish but $\left|H_{z}\right|^{2}$ and $\left|H_{r}\right|^{2}$ are 100 times weaker than $\left|H_{\varphi}\right|^{2}$. The meshing of the structure leads to non per- fectly circular edges and therefore to a non-zero field at these edges.

This good agreement demonstrates, if necessary, the accuracy of the Order-N FDTD spectral method for treating such a problem, and is a very good test of our code. The discrepancy mentioned above allows us to define an absolute precision on the value of $\widetilde{n}$ by $\frac{\Delta \widetilde{n}}{\tilde{n}}=5 \%$.

\section{$4 \quad$ Dispersion relations and modes for a silver structure}

In the following, we replace the perfect metal by silver, which has its dielectric constant described by a Drude model ${ }^{1}$. First, we study the influence of the grating period, $p$, on the values of the eigenfrequencies. The results are plotted in Fig. 6. We can see that each spectrum is more complicated than in the case of a perfect metal. For a perfect metal, only two peaks occurred between $\lambda=200 \mathrm{~nm}$ and $\lambda=800 \mathrm{~nm}$ and their linewidths are very close to zero but do not absolutely vanish because of the time sampling necessary to calculate the spectrum (see reference [14] for more details). In the case of silver, other peaks occur (primarily four peaks or more precisely two bands with double peak features) and their widths do not vanish. This broadening is induced by damping in metal and could be related to the imaginary part of the effective index.

The spectra of Fig. 6 contain two band structures. The position of the UV band, between $300 \mathrm{~nm}$ and $400 \mathrm{~nm}$, remains almost constant when the grating period, $p$, is increased from 160 to $350 \mathrm{~nm}$. This can be interpreted by the fact that

\footnotetext{
${ }^{1}$ The Drude model is given by: $\varepsilon(\omega)=1-\frac{\omega_{p}^{2}}{\omega(\omega+i \gamma)}$. For silver, we set $\omega_{p}=\omega_{p}^{\mathrm{Ag}}=1.374 \times 10^{16} \mathrm{rad} / \mathrm{s}$ and $\gamma=\gamma^{\mathrm{Ag}}=3.21 \times 10^{13} \mathrm{rad} / \mathrm{s}$.
} 


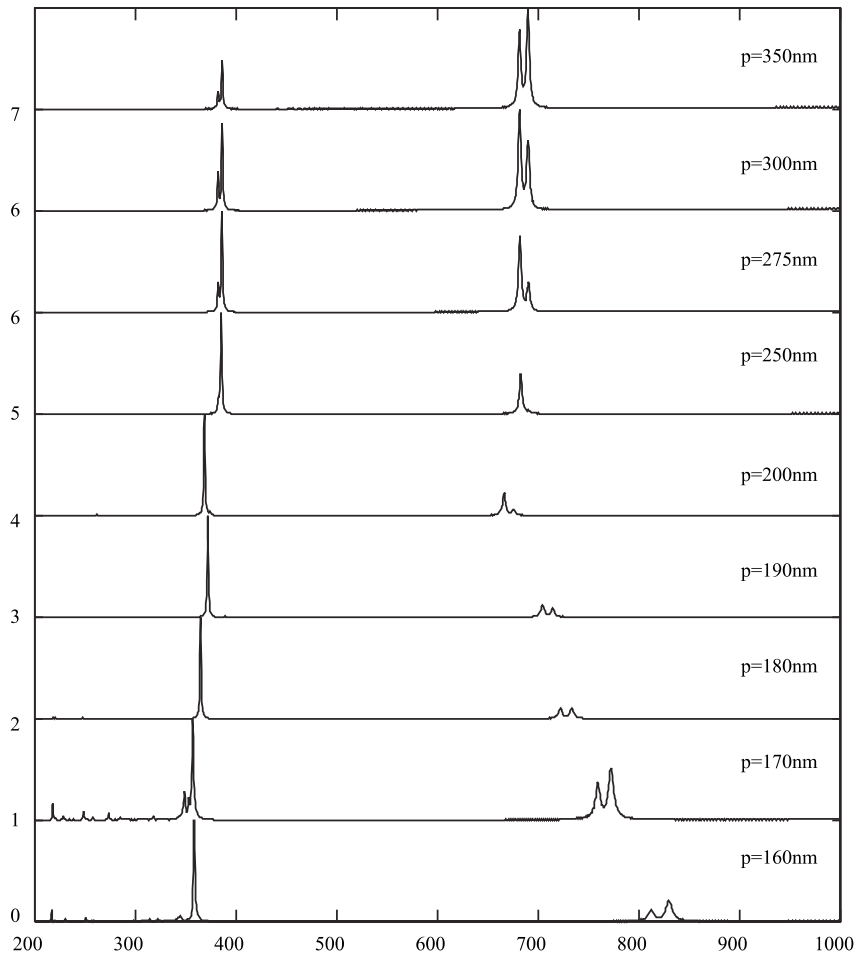

FIGURE 6 Normalized spectral intensities versus the period of a silver coaxial waveguide grating for $k_{z}=0$ (the in-plane modes). The other geometrical parameters of the structure are $a=75 \mathrm{~nm}$ and $b=50 \mathrm{~nm}$ (see Fig. 2b)

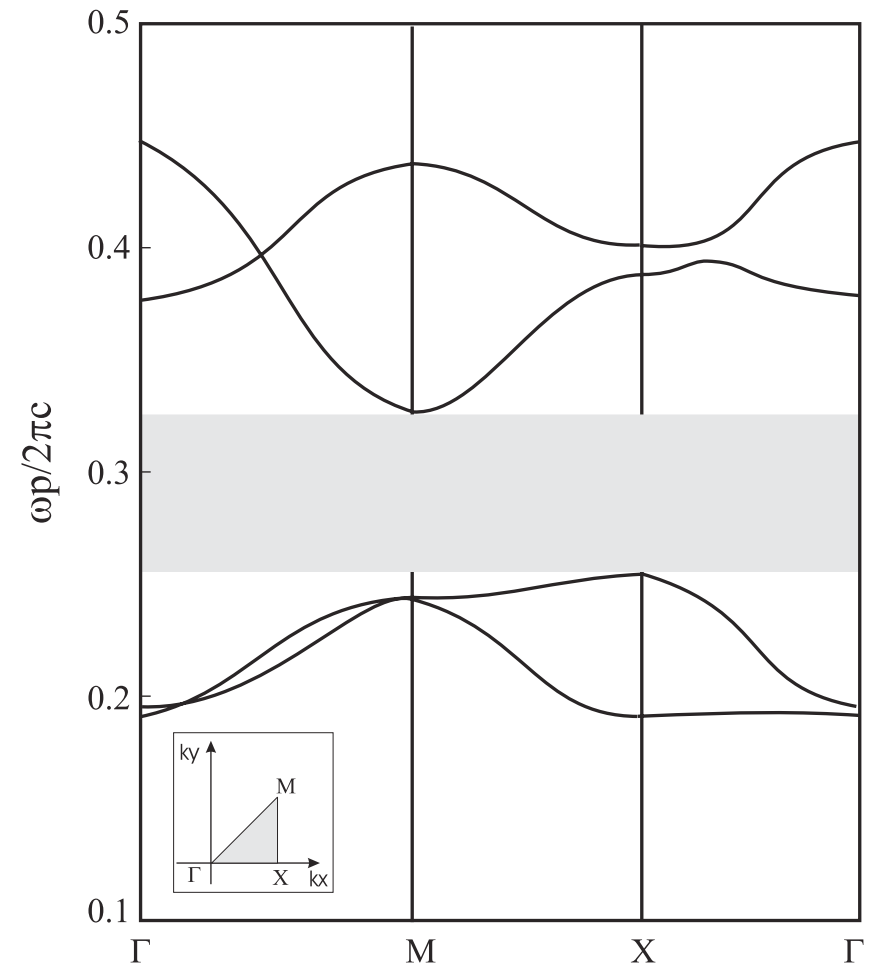

FIGURE 7 In-plane $\left(k_{z}=0\right)$ photonic band structure for $H$ polarization of the silver annular 2-D grating with $a=75 \mathrm{~nm}, b=50 \mathrm{~nm}$ and $p=160 \mathrm{~nm}$

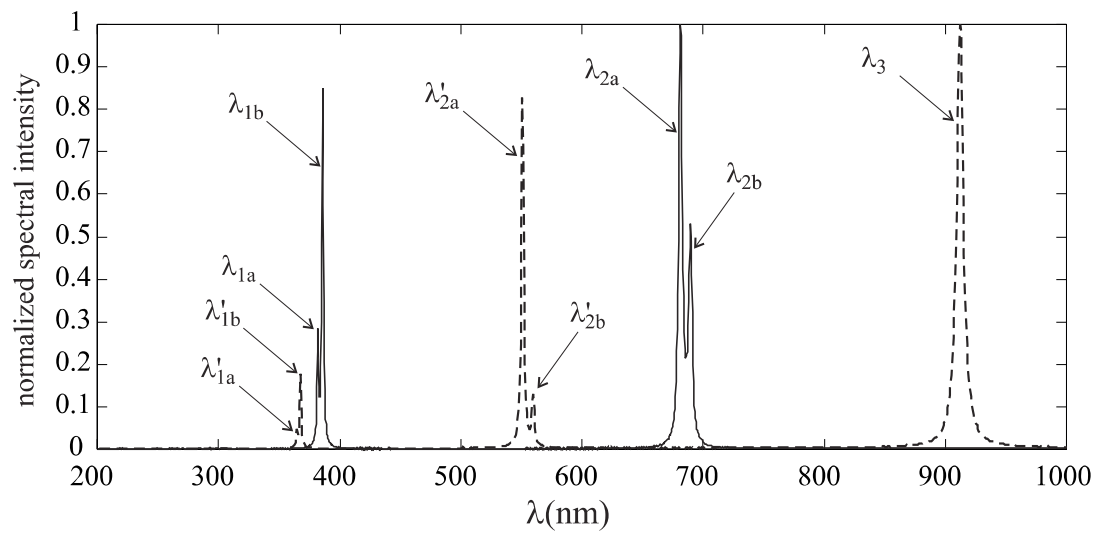

FIGURE 8 Normalized spectral intensities for two different $k_{z}$ values in the case of a silver coaxial waveguide. $k_{z}=0$ for the solid line and $k_{z}=\frac{2 \pi}{500} \mathrm{~nm}^{-1}$ for the dashed line

the spatial distribution of this mode is very confined around the waveguide which induces a weak coupling between two closer waveguides. However, the other band, in the red part of the spectra, has a position which exhibits large variations when the period is increased. This mode seems more sensitive to coupling between cavities. But this band position remains stable when $p \geq 250 \mathrm{~nm}$. It means that for $p=300 \mathrm{~nm}$, which is the period of the AAA structure studied in transmission, the coupling between adjacent coaxial cavities remains small.

Figure 6 shows that for $p=160 \mathrm{~nm}$ the coaxial waveguides are coupled. Therefore, in this case, it is interesting to study the in-plane modes of such a structure when the Bloch vector runs over the first Brillouin zone.

In Fig. 7 we present the photonic band structure for the case of $H$ polarization (TE modes) when $a=75 \mathrm{~nm}, b=$ $50 \mathrm{~nm}$ and $p=160 \mathrm{~nm}$. For this polarization, an absolute

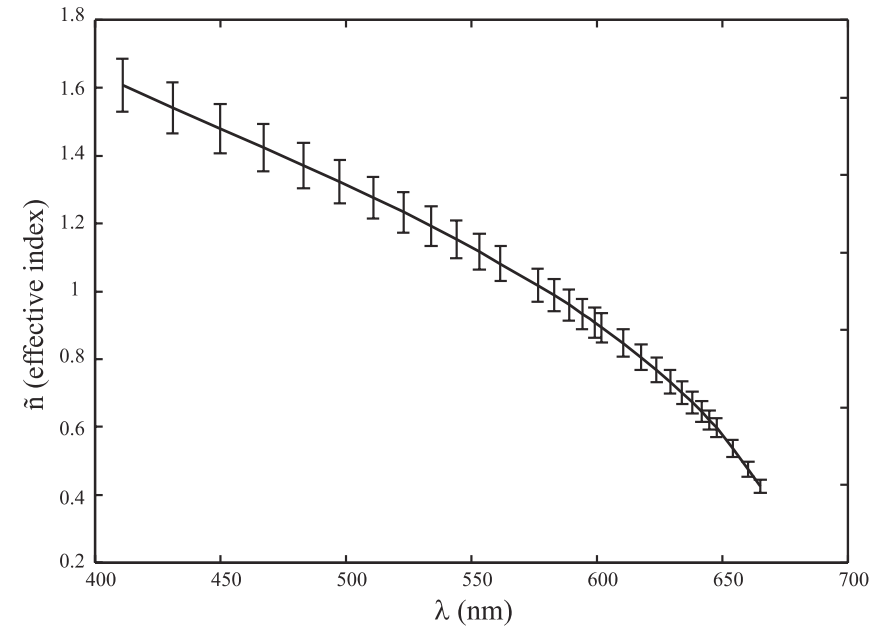

FIGURE 9 Effective index for the $\mathrm{TE}_{1}$ guided mode. Errorbars are indicated with respect to the calculated $\Delta \tilde{n}$ in Sect. 3 

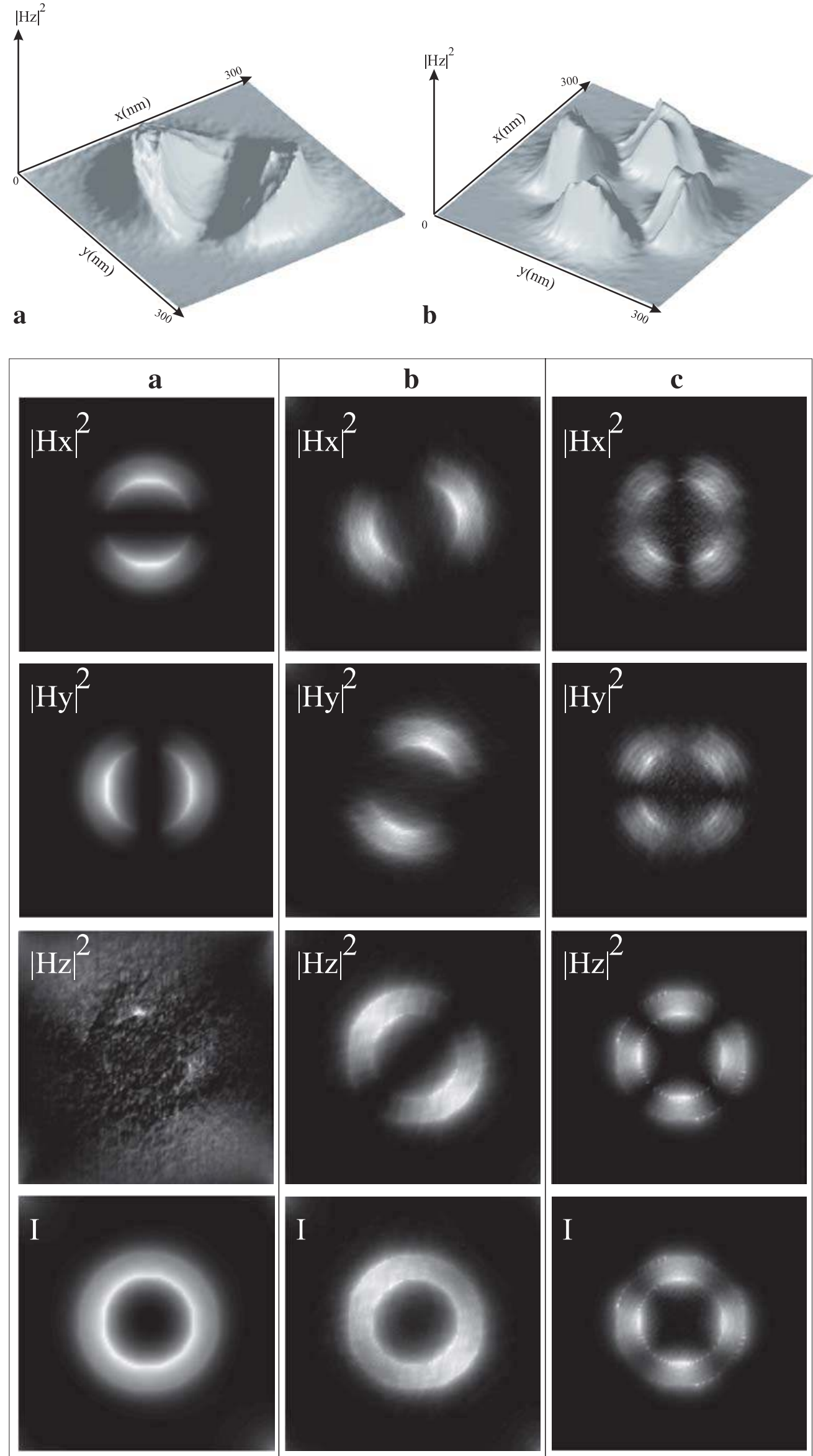

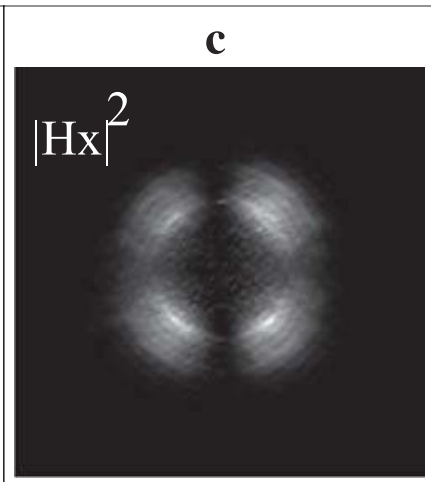

FIGURE $10|h z|^{2}$ of the two inplane modes $\left(k_{z}=0\right)$ of the silver coaxial waveguide. a For $\lambda_{2 a}=$ $681 \mathrm{~nm}$, and $\mathbf{b}$ for $\lambda_{1 b}=385 \mathrm{~nm}$
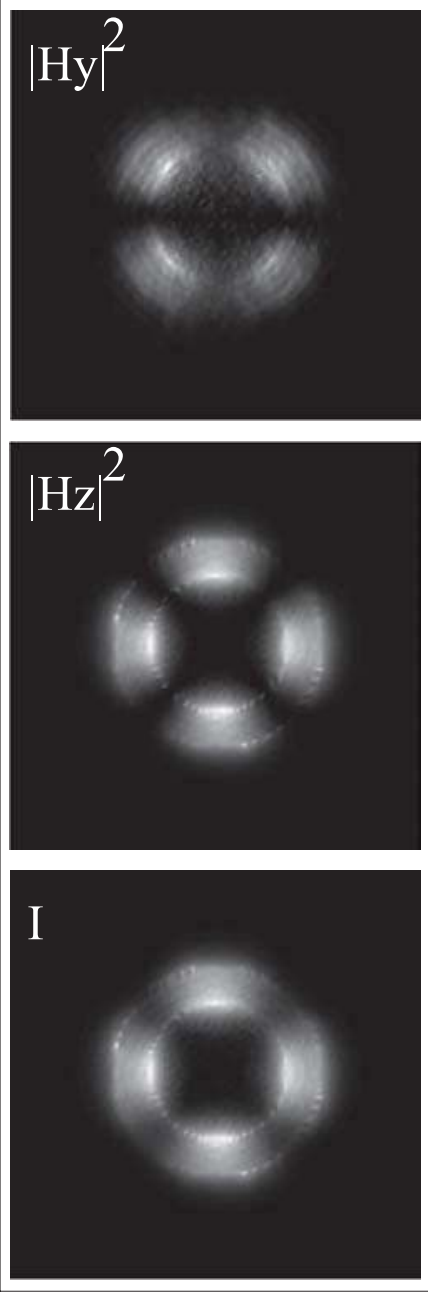

FIGURE 11 Square modulus of the $E_{x}, E_{y}$ and $H_{z}$ components for the three peaks of the dashed line in Fig. 8. a Corresponds to $\lambda_{3}$, b to $\lambda_{1 b}$, and $\mathbf{c}$ to $\lambda_{2 a}^{\prime}$ 
band gap is present in this structure (shaded zone) between $\lambda=492 \mathrm{~nm}$ and $\lambda=630 \mathrm{~nm}$.

Let us now study the grating with $p=300 \mathrm{~nm}$. Figure 8 shows the normalized spectral intensities of the 2-D silver structure for two different values of $k_{z}$.

The solid line of Fig. 8, which is an enlargement of one curve of Fig. 6, corresponds to $k_{z}=0$. Two double peaks appear and are located at $\lambda_{1 a}=382 \mathrm{~nm}$ and $\lambda_{1 b}=386 \mathrm{~nm}$ for the first one and at $\lambda_{2 a}=681 \mathrm{~nm}$ and $\lambda_{2 b}=689.6 \mathrm{~nm}$ for the second one. The double peak structure is discussed below.

When $k_{z}=\frac{2 \pi}{500} \mathrm{~nm}^{-1}$ (dashed line in Fig. 8), the two double peaks are shifted $\left(\lambda_{1 a} \rightarrow \lambda_{1 a}^{\prime}=363.6 \mathrm{~nm} ; \lambda_{1 b} \rightarrow\right.$ $\lambda_{1 b}^{\prime}=366.6 \mathrm{~nm} ; \lambda_{2 a} \rightarrow \lambda_{2 a}^{\prime}=549.7 \mathrm{~nm}$ and $\lambda_{2 b} \rightarrow \lambda_{2 b}^{\prime}=$ $558.7 \mathrm{~nm}$ ). These shifts do not verify equation (2). The effective indices of $\lambda_{2 a}^{\prime}$ and $\lambda_{2 b}^{\prime}$ modes are larger than 1 . The variations in the effective index of this mode versus the wavelength are presented in Fig. 9. These variations are in good agreement with the curve published by Moreau et al. [6] in the case of a square coaxial geometry.

$\widetilde{n}>1$ for $\lambda<570 \mathrm{~nm}$; it is less than 1 near the cutoff and it falls to zero at the cutoff $(\lambda \simeq 700 \mathrm{~nm})$.

A new spectral line appears at $\lambda_{3}=910 \mathrm{~nm}$ in the dashed line of Fig. 8. This latter line is exclusively composed of only one peak and should correspond to a TEM mode for a silver waveguide. Its effective index is equal to 1.82 instead of one for a perfect metal.

Figure 10 show the distributions of $|h z|^{2}$ for two in-plane modes in the case of a silver coaxial waveguide. Figure 10a, calculated for $\lambda_{2 a}$, seems to correspond to a $\mathrm{TE}_{11}$ mode while Fig. 10b, which is calculated for $\lambda_{1 b}$, has the characteristics of a $\mathrm{TE}_{21}$ mode.

In the case of the out-off plane modes and for $k_{z}=$ $\frac{2 \pi}{500} \mathrm{~nm}^{-1}$, we present in Fig. 11 the spatial distribution of the square modulus of the three cartesian components $\left(\left|H_{x}\right|^{2}\right.$, $\left|H_{y}\right|^{2}$ and $\left.\left|H_{z}\right|^{2}\right)$ of the magnetic field and their sum $(I=$ $\left|H_{x}\right|^{2}+\left|H_{y}\right|^{2}+\left|H_{z}\right|^{2}$ ).

The first column (a) corresponds to $\lambda_{3}$. We find that the $H_{z}$ component is zero and $H_{x}$ and $H_{y}$ vary as $\sin \varphi$ and $\cos \varphi$ respectively which corresponds effectively to the angular properties of a $\mathrm{TEM}_{0}$ mode.

The second column (Fig. 11b) and the third one (Fig. 11c) correspond to the two other peaks of the dashed line of Fig. 8 ( $\lambda_{2 a}^{\prime}$ and $\lambda_{1 b}^{\prime}$ respectively). The spatial distribution of the intensities confirms the nature of these two modes; the first one looks like a $\mathrm{TE}_{1}$ mode while the second one is similar to a $\mathrm{TE}_{2}$ one.

\section{Remarks:}

- For a perfect metallic waveguide, $\mathrm{TE}_{m}$ and $\mathrm{TM}_{m}$ modes are independent. In the case of a dielectric and non-perfect metal, pure TE and TM only exist for $m=0$. When $m>0$, all of the modes have $E_{z}$ and $H_{z}$ different from zero, so these modes are named $H E_{m}$ or $E H_{m}$. In our case, we have verified that the $E_{z}$ component of the guided modes remains zero as for the perfect metal. So, in the following, we keep the notations introduced above.

- We have interpreted the main features of the spectra. It remains for us to explain the origin of the fine structure; double peaks appearing in the case of silver (see Fig. 8). For one coaxial waveguide, the physical problem has a cylin- drical symmetry; in this case the direction of the $x$-axis is arbitrary and the $\mathrm{TE}_{1}$ mode is in fact degenerated. This cylindrical symmetry is broken when coaxial cavities are arranged in a square grating. As mentioned above, the coupling between adjacent cavities is very weak but it does not completely vanish and it induces a breaking of the degeneracy. We have verified that the two peaks of the same band are $\mathrm{TE}_{1}$ modes. For a single waveguide $(p \rightarrow \infty)$, only one peak should occur instead of a double structure. The TEM mode has no fine structure because it has a cylindrical symmetry that is not perturbed by the adjacent cavities.

\section{Discussion}

In light of these results, the interpretation of transmission spectra for AAA structures (Fig. 1b and c) for $400 \mathrm{~nm}<\lambda<800 \mathrm{~nm}$ can be clarified. All of the enhanced transmission peaks correspond to a resonance of one mode: the $\mathrm{TE}_{1}$ mode. The $\alpha$-band located around $\lambda=700 \mathrm{~nm}$ corresponds to the cutoff $\left(k_{z}=0\right)$. In this case, the effective index vanishes and this mode does not really propagate. Its position is independent of metal thickness, and it appears in all of the spectra.

In order to verify this assumption, in Fig. 12 we present the cross-sections of the field intensity in the $x z$ plane $(e=300 \mathrm{~nm})$ for the three bands of the spectrum presented in Fig. 1b. We note that the $\alpha$-band intensity is pretty constant along $z$ inside the cavity. The $\beta$-band has one node located at the middle of the cavity, while there are two nodes for the $\delta$-band. At resonances, a standing wave structure is created in the cavities with maxima at the edges.

It is remarkable that the peak positions (except for the $\alpha$ one) in the transmission spectra of one AAA structure can be described by one simple equation that is valid for any
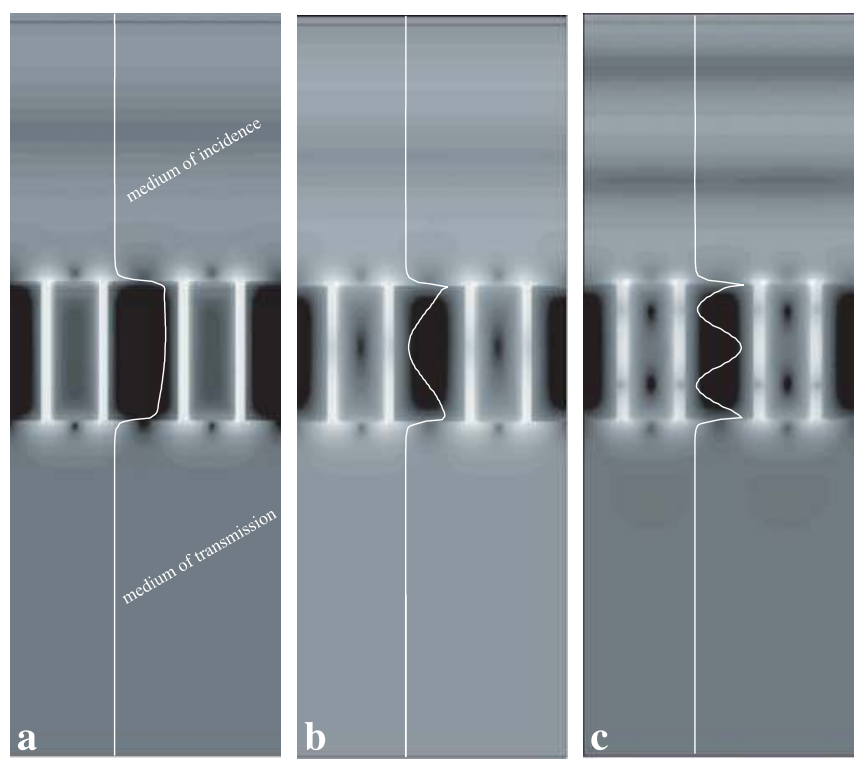

FIGURE 12 Cross-sections of field intensities in logarithmic gray scale. a In the case of the $\alpha$-band, $\mathbf{b}$ for the $\beta$-band, and $\mathbf{c}$ for the $\delta$-band. The structure parameters are given in Fig. 1a, with $e=300 \mathrm{~nm}$ 
thickness:

$\frac{4 \pi \tilde{n}(\lambda) e}{\lambda}+2 \varphi_{r}=2 l \pi$

where $l$ is a positive integer and $\varphi_{r}$ is the phase of the reflection coefficient of the cavity's sides. $\widetilde{n}(\lambda)$ is the effective index determined above (Fig. 9) for an infinite photonic crystal. $\varphi_{r}$ is reasonably independent of the film thickness $\left.\left(\varphi_{r}\right) \simeq 50^{\circ}\right)$.

Note that the TEM and the $\mathrm{TE}_{21}$ modes cannot be excited with a linearly polarized incident beam.

6

\section{Conclusion}

In this paper we have established that the transmission enhancement obtained by a AAA structure is a cavity resonance due to a single guided mode through the coaxial cavities. This interpretation is very similar to the one proposed by Lalanne et al. $[3,16]$ in the case of a 1-D array. The "horizontal" surface plasmons, along the $(x-y)$ plane, are not the origin of the enhanced transmission in the case of the AAA structure.

The dispersion properties of the real metal are of great importance to inducing the transmission enhancement. The wavelength cutoff is shifted from $415 \mathrm{~nm}$ in the case of a perfect metal to $700 \mathrm{~nm}$ for silver for the same geometrical parameters. This value of the cutoff wavelength depends both on the nature of the metal (the dielectric constant) and on the geometry of the object.

The guided mode is excited by diffraction at the input side of the metallic film. Because of the linear polarization of the incident beam, the "TEM" mode cannot be excited. However, it could be generated by using a radially polarized Bessel beam [17]. In this case, enhanced transmission could be expected at a larger wavelength $(\lambda \simeq 900 \mathrm{~nm}$ see Fig. 8$)$.

\section{REFERENCES}

1 T.W. Ebbesen, H.J. Lezec, H.F. Ghaemi, T. Thio, P.A. Wolff: Nature 391, 667 (1998)

2 F. Baida, D. Van Labeke: Opt. Commun. 209, 17 (2002)

3 S. Astilean, P. Lalanne, M. Palamaru: Opt. Commun. 175, 265 (2000)

4 A. Krishnan, T. Thio, T.J. Kim, H.J. Lezec, T.W. Ebbesen, P.A. Wolff, J. Pendry, L. Martin-Moreno, F.J. Garcia-Vidal: Opt. Commun. 200, 1 (2001)

5 F.I. Baida, D. Van Labeke: Phys. Rev. B 67, 155314 (2003)

6 A. Moreau, G. Granet, F.I. Baida, D. Van Labeke: Opt. Express 11-(10), 1131 (2003)

7 T. Thio, H.J. Lezec, T.W. Ebbesen: Physica B 279, 90 (2000)

8 T.J. Kim, T. Thio, T.W. Ebessen, D.E. Grupp, H.J. Lezec: Opt. Lett. 24, 256 (1999)

9 R.J. Blaikie, S.J. McNab: Appl. Opt. 40, 1692 (2001)

10 L. Martin-Moreno, F.J. Garcia-Vidal, H.J. Lezec, K.M. Pellerin, T. Thio, J.B. Pendry, T.W. Ebbesen: Opt. Lett. 86, 1114 (2001)

11 K. Sakoda, N. Kawai, T. Ito, A. Chutinan, S. Noda, T. Mitsuyu, K. Hirao: Phys. Rev. B 64, 045116 (2001)

12 K. Sakoda: Optical Properties of Photonic Crystals (Springer, Berlin Heidelberg New York 2001)

13 A. Taflove, S. Hagness: Computational Electrodynamics: The FiniteDifference Time-Domain Method., 2nd Edn (Artech House, Boston, MA 2000)

14 C.T. Chan, Q.L. Yu, K.M. Ho: Phys. Rev. B 51, 16635 (1995)

15 A.W. Snyder, J. D. Love: Optical Waveguide Theory (Chapman \& Hall, London 1983)

16 P. Lalanne, J.P. Hugonin, S. Astilean, M. Palamaru, K.D. Möller: J. Opt. A: Pure Appl. Opt. 2, 48 (2000)

17 T. Grosjean, D. Courjon, M. Spajer: Opt. Commun. 203, 1 (2002) 